\title{
Engineering Notes
}

ENGINEERING NOTES are short manuscripts describing new developments or important results of a preliminary nature. These Notes should not exceed 2500 words (where a figure or table counts as 200 words). Following informal review by the Editors, they may be published within a few months of the date of receipt. Style requirements are the same as for regular contributions (see inside back cover).

\section{Global Directional Control of a Slender Autonomous Underwater Vehicle}

\author{
Hye-Young Kim \\ Angstrom, Inc., Seongnam, Republic of Korea \\ and \\ Craig A. Woolsey \\ Virginia Polytechnic Institute and State University, \\ Blacksburg, Virginia 24061-0203
}

DOI: $10.2514 / 1.20475$

\section{Introduction}

$\mathbf{C}$ ONVENTIONAL streamlined autonomous underwater vehicles (AUVs) are underactuated by design. Typically, they include a propulsor and torque actuators, such as control planes. In this Note, it is assumed that the 6 degrees of freedom vehicle is actuated in 4 degrees of freedom: surge, roll, pitch, and yaw. The control objective is to globally asymptotically stabilize longitudinalaxis translation of the vehicle in a desired inertial direction. A provably convergent, global directional controller could enhance the operation of agile vehicles in dynamic environments, such as rivers, tidal basins, or other coastal areas. Energy-based tools are appealing for designing such controllers because they provide natural candidate Lyapunov functions for stability analysis. As an example, a passivity-based technique known as interconnection and damping assignment was used to stabilize the unstable dynamics of a slender, underactuated AUV in [1]. The paper assumed an idealized (inviscid) dynamic model and did not consider the attitude kinematics. A nonlinear directional controller was developed in [2] using potential energy shaping, although that model also omitted viscous effects. This Note treats the directional stabilization problem for a vehicle model which requires minimal assumptions about the viscous effects. The result therefore allows a broad range of viscous force and moment models.

Directional stabilization for AUVs may be considered a natural, intermediate step between waypoint navigation and general, threedimensional path following, as described in [3] , for example. Several approaches have been proposed to address three-dimensional path following for underactuated, 6 degrees of freedom AUVs. These approaches include adaptive feedback [4], switching theory [5], and backstepping $[3,6]$. Here, we consider the more fundamental problem of directional stabilization for a slender, underactuated AUV and we approach the problem from an energy perspective. The

Received 11 October 2005; accepted for publication 11 August 2006. Copyright $@ 2006$ by the American Institute of Aeronautics and Astronautics, Inc. All rights reserved. Copies of this paper may be made for personal or internal use, on condition that the copier pay the $\$ 10.00$ per-copy fee to the Copyright Clearance Center, Inc., 222 Rosewood Drive, Danvers, MA 01923; include the code $\$ 10.00$ in correspondence with the CCC.

*Optical System Engineer, Former Post-Doctoral Research Assistant, Virginia Tech's Aerospace and Ocean Engineering Department; gpdud@yahoo.com.

${ }^{\dagger}$ Associate Professor, Department of Aerospace and Ocean Engineering; cwoolsey@vt.edu. stabilization result, which is adapted from [7], is based on the method of feedback passivation described in [ㅇ] . In this approach, a given system is transformed into a feedback interconnection of two passive subsystems. It follows from passive system theory that the interconnection is passive and therefore that the system is stable. Asymptotic stability may be shown through further analysis, with suitably defined feedback dissipation included, if necessary. As is the case in this Note, exploiting intrinsic properties such as passivity often allows one to derive control algorithms which are globally effective and which work with the natural dynamics rather than dominate or supplant them.

\section{AUV Equations of Motion}

The AUV is modeled as a slender, axisymmetric rigid body whose mass $m$ equals the mass of the fluid which it displaces; thus, the vehicle is neutrally buoyant. The vehicle is equipped with a single propulsor, which is aligned with the axis of symmetry, and with torque actuators that provide independent control moments about all three axes.

Vehicle kinematics: The principal axes of the displaced fluid define a body-fixed reference frame represented by the unit vectors $\boldsymbol{b}_{1}, \boldsymbol{b}_{2}$, and $\boldsymbol{b}_{3}$ in Fig. 1 . Another reference frame, denoted by the unit vectors $\boldsymbol{i}_{1}, \boldsymbol{i}_{2}$, and $\boldsymbol{i}_{3}$, is fixed in inertial space. The location of the body frame with respect to the inertial frame is given by the inertial vector $x$. The body's orientation with respect to inertial space is given by the unit quaternion $\boldsymbol{q}=\left[q_{0}, \boldsymbol{q}_{\chi}^{T}\right]^{T}$. The unit quaternions provide a global, if redundant, parameterization of vehicle attitude; see [9] for a general review of attitude parameterizations and [10] for an early application of quaternions for underwater vehicle control.

Let $\boldsymbol{v}=\left[v_{1}, v_{2}, v_{3}\right]^{T}$ represent the translational velocity and let $\boldsymbol{\omega}=\left[\omega_{1}, \omega_{2}, \omega_{3}\right]^{T}$ represent the rotational velocity of the AUV with respect to inertial space, where $v$ and $\omega$ are both expressed in the body frame. Also, define the operator $\hat{\bullet}$ such that for any 3 -vectors $\boldsymbol{a}$ and $\boldsymbol{b}, \hat{\boldsymbol{a}} \boldsymbol{b}=\boldsymbol{a} \times \boldsymbol{b}$. The $3 \times 3$ skew-symmetric matrix $\hat{\boldsymbol{a}}$ is sometimes called the "cross-product equivalent" matrix corresponding to the vector $\boldsymbol{a}$. The kinematic equations are

$$
\begin{aligned}
& \dot{\boldsymbol{x}}=\boldsymbol{R}_{\mathrm{IB}}(\boldsymbol{q}) \boldsymbol{v} \\
& \dot{\boldsymbol{q}}=\frac{1}{2} \boldsymbol{Q}(\boldsymbol{q}) \boldsymbol{\omega}
\end{aligned}
$$

where

$$
\boldsymbol{R}_{\mathrm{IB}}(\boldsymbol{q})=\mathbb{1}-2\left(q_{0} \mathbb{1}-\hat{\boldsymbol{q}}_{\chi}\right) \hat{\boldsymbol{q}}_{\chi} \quad \text { and } \quad \boldsymbol{Q}(\boldsymbol{q})=\left[\begin{array}{c}
-\boldsymbol{q}_{\chi}^{T} \\
q_{0} \mathbb{1}+\hat{\boldsymbol{q}}_{\chi}
\end{array}\right]
$$

and where $\mathbb{1}$ is the $3 \times 3$ identity matrix.

Vehicle dynamics: Having assumed, without further loss of generality, that the 1 -axis is the axis of symmetry, define the diagonal matrix $\boldsymbol{M}_{11}=\operatorname{diag}\left(m_{1}, m_{2}, m_{2}\right)$ as the sum of $m \mathbb{1}$ and the added mass matrix. Similarly, let $\boldsymbol{M}_{22}$ represent the sum of the rigid body inertia and the diagonal added inertia matrix. Finally, suppose that the center of mass is located by the body frame vector $\boldsymbol{r}_{\mathrm{cm}}$ and let $\boldsymbol{M}_{12}=-m \hat{\boldsymbol{r}}_{\mathrm{cm}}$. Then $\boldsymbol{v}$ and $\boldsymbol{\omega}$ can be related to the body/fluid linear momentum $\boldsymbol{p}$ and the body/fluid angular momentum $\boldsymbol{h}$ as follows:

$$
\left(\begin{array}{l}
\boldsymbol{p} \\
\boldsymbol{h}
\end{array}\right)=\left(\begin{array}{ll}
\boldsymbol{M}_{11} & \boldsymbol{M}_{12} \\
\boldsymbol{M}_{12}^{T} & \boldsymbol{M}_{22}
\end{array}\right)\left(\begin{array}{c}
\boldsymbol{v} \\
\boldsymbol{\omega}
\end{array}\right)
$$




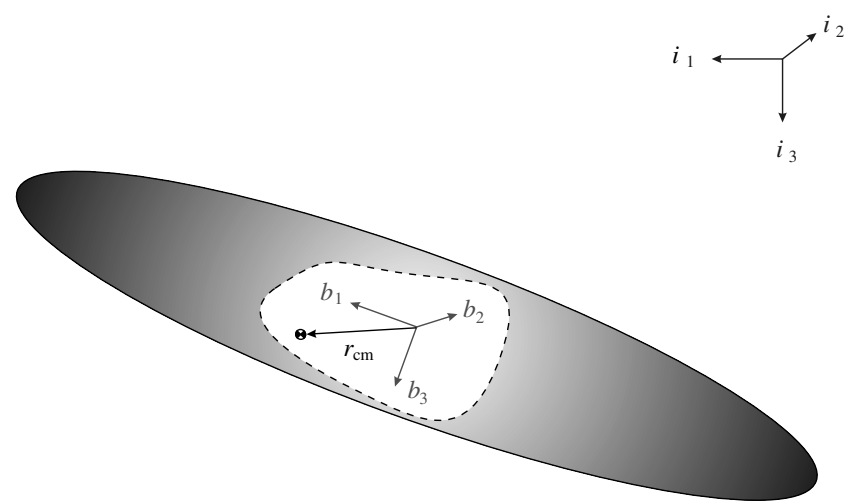

Fig. 1 Spheroidal underwater vehicle.

More precisely, $\boldsymbol{p}$ and $\boldsymbol{h}$ represent the translational and rotational impulse required to instantaneously set the body into motion through the fluid at velocity $\boldsymbol{v}$ and angular rate $\boldsymbol{\omega}$; see [11]. In any case, $\boldsymbol{p}$ and $\boldsymbol{h}$ evolve according to a finite system of ordinary differential equations which describe the body's motion. Let $\boldsymbol{f}_{v}$ and $\boldsymbol{\tau}_{v}$ represent the viscous force and moment, respectively, and let $f_{c}$ and $\boldsymbol{\tau}_{c}$ represent thrust and the control moment. Defining $\boldsymbol{\beta}_{1}=[1,0,0]^{T}$, the AUV dynamic equations are

$$
\begin{gathered}
\dot{\boldsymbol{p}}=\boldsymbol{p} \times \boldsymbol{\omega}+\boldsymbol{f}_{v}+f_{c} \boldsymbol{\beta}_{1} \\
\dot{\boldsymbol{h}}=\boldsymbol{h} \times \boldsymbol{\omega}+\boldsymbol{p} \times \boldsymbol{v}+\boldsymbol{r}_{\mathrm{cm}} \times m g\left(\boldsymbol{R}_{\mathrm{IB}}^{T} \boldsymbol{i}_{3}\right)+\boldsymbol{\tau}_{v}+\boldsymbol{\tau}_{c}
\end{gathered}
$$

Some remarks on the dynamic equations (4) and (5) are in order.

1) Because $\boldsymbol{M}_{11}$ is not a scalar multiple of the identity, the linear momentum of an underwater vehicle is generally not collinear with its linear velocity. This is an important distinction from heavier-thanair flight vehicles and spacecraft which accounts, for example, for the "Munk moment" appearing in the term $\boldsymbol{p} \times \boldsymbol{v}$ in (5); see [12].

2) While there is no net gravitational force, because the vehicle is assumed to be neutrally buoyant, a gravitational moment $\boldsymbol{r}_{\mathrm{cm}} \times$ $m g\left(\boldsymbol{R}_{\mathrm{IB}}^{T} \boldsymbol{i}_{3}\right)$ appears on the right side of Eq. (5). This "restoring moment" tends to keep the body's center of mass below its center of buoyancy, unless the moment is countered by the control moment $\boldsymbol{\tau}_{c}$.

The terms $\boldsymbol{f}_{v}(\boldsymbol{v}, \boldsymbol{\omega})$ and $\boldsymbol{\tau}_{v}(\boldsymbol{v}, \boldsymbol{\omega})$ represent the viscous force and moment, respectively, that act on the vehicle as a consequence of its motion through the fluid. We assume quasisteady flow, as is standard for air and marine vehicle dynamic models. Even under the quasisteady flow assumption, however, $\boldsymbol{f}_{v}$ and $\boldsymbol{\tau}_{v}$ are difficult to express explicitly over the full range of motion. Rather than restrict the model's validity to a small neighborhood of the nominal motion, we make some simple and relatively general modeling assumptions.

Assumption 2.1.

$$
\begin{gathered}
\boldsymbol{\tau}_{v} \cdot \boldsymbol{\omega} \leq 0 \quad \text { when } \boldsymbol{\omega} \neq \mathbf{0} \\
\boldsymbol{\tau}_{v}=\mathbf{0} \quad \text { when }(\boldsymbol{\omega}, \boldsymbol{v})=\left(\mathbf{0}, v \boldsymbol{\beta}_{1}\right) \quad \forall v \in \mathbb{R}
\end{gathered}
$$

In words, we assume that the viscous moment opposes the angular rate, in general, and that it vanishes for pure translation along the longitudinal axis.

We impose a bit more structure on the model of the viscous force. By definition, drag opposes vehicle velocity and lift acts orthogonally to the velocity vector. Considering axial symmetry, we assume that the lift force acts in the plane determined by the velocity vector and the vehicle's longitudinal axis. This assumption fails to capture out-of-plane forces due to asymmetric fluid flow, however, it is consistent with standard modeling assumptions. We also neglect the dependence of $\boldsymbol{f}_{v}$ on $\boldsymbol{\omega}$, but we note in Remark 3.2 that our approach could accommodate such dependence, in certain cases.

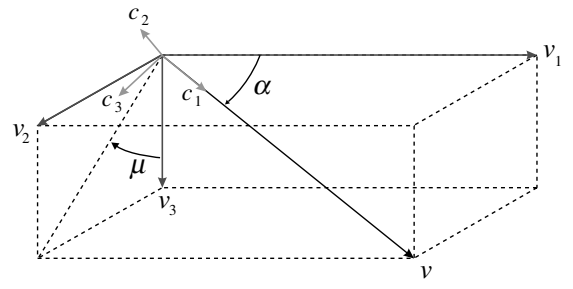

Fig. 2 Hydrodynamic angles for an axisymmetric body.

To express $\boldsymbol{f}_{v}(\boldsymbol{v})$, we introduce two hydrodynamic angles. Let

$$
\mu= \begin{cases}\arctan \left(\frac{v_{2}}{v_{3}}\right) & v_{2} \neq 0 \quad \text { and } / \text { or } \quad v_{3} \neq 0 \\ 0 & v_{2}=v_{3}=0\end{cases}
$$

where the "4-quadrant" arctangent is used. Identifying $\pi$ with $-\pi$, we have $\mu \in(-\pi, \pi]$. Rotating the body frame through the angle $\mu$ about the $\boldsymbol{b}_{1}$ axis defines an intermediate reference frame in which the velocity vector has components only in the intermediate frame's " 1 3" plane. Moreover, the 3 -axis component of velocity in this intermediate frame is nonnegative. Let

$$
\alpha= \begin{cases}\arctan \left(\frac{\sqrt{v_{2}^{2}+v_{3}^{2}}}{v_{1}}\right) & \boldsymbol{v} \neq \mathbf{0} \\ 0 & \boldsymbol{v}=\mathbf{0}\end{cases}
$$

where, once again, the 4-quadrant arctangent is used. Note that $\alpha \in[0, \pi]$. Rotating through the angle $\alpha$ about the intermediate 2 -axis yields a new reference frame, defined by unit vectors $c_{1}, c_{2}$, and $c_{3}$, in which the 1-axis is aligned with the velocity vector as shown in Fig. 2. Following the terminology of [13], we refer to this reference frame as the "current frame." The proper rotation matrix

$$
\begin{aligned}
& \boldsymbol{R}_{\mathrm{BC}}(\mu, \alpha)=e^{-\mu \hat{\boldsymbol{\beta}}_{1}} e^{-\alpha \hat{\boldsymbol{\beta}}_{2}} \\
& \quad=\left(\begin{array}{ccc}
\cos \alpha & 0 & -\sin \alpha \\
\sin \mu \sin \alpha & \cos \mu & \sin \mu \cos \alpha \\
\cos \mu \sin \alpha & -\sin \mu & \cos \mu \cos \alpha
\end{array}\right)
\end{aligned}
$$

transforms free vectors from the current frame to the body frame.

Remark 2.2: The hydrodynamic angles $\alpha$ and $\mu$ are not the standard angle of attack and sideslip angle; however, the given definitions are more convenient for this work. Note that $\alpha$ is discontinuous when $\boldsymbol{v}=\mathbf{0}$ and that $\mu$ is discontinuous when $\alpha=0$. Thus, $\boldsymbol{R}_{\mathrm{BC}}(\mu, \alpha)$ is discontinuous under these conditions. Of course, the discontinuities are an artifact of the definition of the current frame and do not represent any physical effect. Still, special care must be taken in the stability analysis; in fact, the discontinuities are easily treated using Filippov's theory [14] and some extensions described in [15].

It is standard practice to express lift and drag in terms of nondimensional coefficients. To nondimensionalize the forces, we define $f_{0}(\boldsymbol{v})$ as the product of dynamic pressure and a reference area $S$ :

$$
f_{0}(\boldsymbol{v})=\frac{1}{2} \rho\|\boldsymbol{v}\|^{2} S
$$

where $\rho$ is the fluid density. The viscous force takes the form

$$
\boldsymbol{f}_{v}(\boldsymbol{v})=-f_{0}(\boldsymbol{v}) \boldsymbol{R}_{\mathrm{BC}}(\mu, \alpha)\left(\begin{array}{c}
C_{D}(\alpha) \\
0 \\
C_{L}(\alpha)
\end{array}\right)
$$

We make the following assumptions about the form of the drag and lift coefficients.

Assumption 2.3.

1) $C_{D}(\alpha)$ is a continuous, even function which is positive for all $\alpha$.

2) $C_{L}(\alpha)$ is a continuous, odd function which is positive (negative) when $e^{i \alpha}$ lies in the first or third (second or fourth) quadrant of the complex plane. 
In general, the dimensionless coefficients $C_{D}$ and $C_{L}$ depend on Reynolds number as well as angle of attack. This effect can be incorporated with little impact on the assumptions and the conclusions, but we ignore Reynolds number effects in this presentation.

For a slender, axisymmetric rigid body, the assumption that $C_{D}$ is even and positive is an empirical fact. The assumption regarding the form of $C_{L}$ is consistent with intuition for a slender body in a steady flow. In reality, at large angles of attack, such a body is subject to complicated, unsteady forces that are not captured by simple models; see [16], for example. While these effects certainly impact the dynamics, they are difficult to model accurately. Such effects are typically ignored in control design with the understanding that welldesigned model-based feedback can provide suitable system performance even when the model is imperfect.

\section{Global Asymptotic Directional Stabilization}

The main objective is to stabilize the steady motion

$$
\boldsymbol{q}_{\mathrm{eq}}=\boldsymbol{q}_{d}, \quad \boldsymbol{\omega}_{\mathrm{eq}}=\mathbf{0}, \quad \boldsymbol{v}_{\mathrm{eq}}=\boldsymbol{v}_{d}
$$

where $\boldsymbol{q}_{d}$ is a unit quaternion representing some constant, desired attitude and $\boldsymbol{v}_{d}=v_{d} \boldsymbol{\beta}_{1}$ where $v_{d}>0$ is a constant desired speed. We will first transform the problem, so that the equilibrium is at the origin.

Let $\overline{\boldsymbol{q}}_{d}$ represent the quaternion conjugate of $\boldsymbol{q}_{d}$ and define the attitude error quaternion

$$
\boldsymbol{e}=\left(\begin{array}{c}
e_{0} \\
\boldsymbol{e}_{\chi}
\end{array}\right)=\overline{\boldsymbol{q}}_{d} * \boldsymbol{q}
$$

where $*$ denotes quaternion multiplication; see [9], for example. When $\boldsymbol{q}=\boldsymbol{q}_{d}$, we have $\boldsymbol{e}=\boldsymbol{e}_{d}=[1,0,0,0]^{T}$. To shift the equilibrium to the origin, define the attitude error vector

$$
\tilde{\boldsymbol{e}}=\boldsymbol{e}-\boldsymbol{e}_{d}
$$

Also, define the velocity and corresponding momentum error vectors

$$
\begin{gathered}
\left(\begin{array}{c}
\tilde{\boldsymbol{v}} \\
\tilde{\boldsymbol{\omega}}
\end{array}\right)=\left(\begin{array}{c}
\boldsymbol{v}-\boldsymbol{v}_{\mathrm{eq}} \\
\boldsymbol{\omega}-\omega_{\mathrm{eq}}
\end{array}\right)=\left(\begin{array}{c}
\boldsymbol{v}-\boldsymbol{v}_{d} \\
\boldsymbol{\omega}
\end{array}\right) \text { and } \\
\left(\begin{array}{c}
\tilde{\boldsymbol{p}} \\
\tilde{\boldsymbol{h}}
\end{array}\right)=\left(\begin{array}{ll}
\boldsymbol{M}_{11} & \boldsymbol{M}_{12} \\
\boldsymbol{M}_{12}^{T} & \boldsymbol{M}_{22}
\end{array}\right)\left(\begin{array}{c}
\tilde{\boldsymbol{v}} \\
\tilde{\boldsymbol{\omega}}
\end{array}\right)
\end{gathered}
$$

The complete error dynamics are

$$
\begin{aligned}
& \dot{\tilde{\boldsymbol{e}}}=\frac{1}{2} \boldsymbol{Q}\left(\tilde{\boldsymbol{e}}+\boldsymbol{e}_{d}\right) \tilde{\boldsymbol{\omega}} \\
& \dot{\tilde{\boldsymbol{p}}}=\left(\tilde{\boldsymbol{p}}+\boldsymbol{p}_{d}\right) \times \tilde{\boldsymbol{\omega}}+\boldsymbol{f}_{v}+f_{c} \boldsymbol{\beta}_{1} \\
& \dot{\tilde{\boldsymbol{h}}}=\left(\tilde{\boldsymbol{h}}+\boldsymbol{h}_{d}\right) \times \tilde{\boldsymbol{\omega}}+\left(\tilde{\boldsymbol{p}}+\boldsymbol{p}_{d}\right) \times\left(\tilde{\boldsymbol{v}}+\boldsymbol{v}_{d}\right) \\
& +\boldsymbol{r}_{\mathrm{cm}} \times m g\left(\boldsymbol{R}_{\mathrm{IB}}^{T} \boldsymbol{i}_{3}\right)+\boldsymbol{\tau}_{v}+\boldsymbol{\tau}_{c}
\end{aligned}
$$

The revised control objective is to stabilize the equilibrium at the origin for Eqs. (9-11).

By defining the thrust and control moments appropriately, Eqs. (10) and (11) may be transformed so that they form a passive map from the control moment (the passive input) to the body angular rate (the passive output). The rotational kinematics (9) are well known to be passive. Moreover, the complete equations ( $9-11)$ may be written in a nonlinear cascade form which is amenable to the method of feedback passivation described in []]

Consider the feedback control law

$$
f_{c}=f_{0}(v)\left[\cos \alpha C_{D}(\alpha)-\sin \alpha C_{L}(\alpha)\right]-k_{v_{1}} \tilde{v}_{1}
$$

and

$$
\boldsymbol{\tau}_{c}=-\left[\boldsymbol{r}_{\mathrm{cm}} \times m g\left(\boldsymbol{R}_{\mathrm{IB}}^{T} \boldsymbol{i}_{3}\right)+\boldsymbol{p} \times \boldsymbol{v}_{d}\right]+\boldsymbol{u}
$$

where $k_{v_{1}}>0$ is a control gain and $\boldsymbol{u}$ is a control moment which remains to be determined. The first term in (12) requires that thrust balance the longitudinal component of viscous force and the second term ensures convergence to the desired speed.

In the language of passive systems theory [8], consider the "storage function"

$$
S_{\mathrm{dyn}}=\frac{1}{2}\left(\begin{array}{c}
\tilde{\boldsymbol{p}} \\
\tilde{\boldsymbol{h}}
\end{array}\right)^{T}\left(\begin{array}{ll}
\boldsymbol{M}_{11} & \boldsymbol{M}_{12} \\
\boldsymbol{M}_{12}^{T} & \boldsymbol{M}_{22}
\end{array}\right)^{-1}\left(\begin{array}{c}
\tilde{\boldsymbol{p}} \\
\tilde{\boldsymbol{h}}
\end{array}\right)
$$

Under the given choice of feedback,

$$
\dot{S}_{\mathrm{dyn}}=\tilde{\boldsymbol{v}} \cdot\left(\boldsymbol{f}_{c}+\boldsymbol{f}_{v}\right)+\tilde{\boldsymbol{\omega}} \cdot \boldsymbol{u}+\tilde{\boldsymbol{\omega}} \cdot \boldsymbol{\tau}_{v}
$$

The last term is nonpositive by Assumption 2.1. The first term satisfies

$$
\begin{aligned}
\tilde{\boldsymbol{v}} & \cdot\left(\boldsymbol{f}_{c}+\boldsymbol{f}_{v}\right)=-f_{0}(\boldsymbol{v})\left(\begin{array}{c}
0 \\
\tilde{v}_{2} \\
\tilde{v}_{3}
\end{array}\right)^{T} \boldsymbol{R}_{\mathrm{BC}}(\mu, \alpha)\left(\begin{array}{c}
C_{D}(\alpha) \\
0 \\
C_{L}(\alpha)
\end{array}\right) \\
& +\tilde{v}_{1}\left[f_{c}-f_{0}(\boldsymbol{v})\left(\begin{array}{l}
1 \\
0 \\
0
\end{array}\right) \boldsymbol{R}_{\mathrm{BC}}(\mu, \alpha)\left(\begin{array}{c}
C_{D}(\alpha) \\
0 \\
C_{L}(\alpha)
\end{array}\right)\right] \\
& =-\left(\tilde{v}_{2} \sin \mu+\tilde{v}_{3} \cos \mu\right)\left[\sin \alpha C_{D}(\alpha)+\cos \alpha C_{L}(\alpha)\right] f_{0}(\boldsymbol{v}) \\
& +\tilde{v}_{1}\left\{f_{\mathrm{c}}-f_{0}(\boldsymbol{v})\left[\cos \alpha C_{D}(\alpha)-\sin \alpha C_{L}(\alpha)\right]\right\}
\end{aligned}
$$

Consider the first term in (16). By definition of the angle $\mu$,

$$
\tilde{v}_{2} \sin \mu+\tilde{v}_{3} \cos \mu=\sqrt{\tilde{v}_{2}^{2}+\tilde{v}_{3}^{2}} \geq 0
$$

Also, given the assumptions on the form of $C_{D}$ and $C_{L}$ and the fact that $\alpha \in[0, \pi]$, we have

$$
\sin \alpha C_{D}(\alpha)+\cos \alpha C_{L}(\alpha) \geq 0
$$

[and strictly positive for $\alpha \in(0, \pi)$ ]. Therefore, the first term in $(\underline{16})$ is nonpositive. Now consider the second term in (16) and define $f_{c}$ according to (12). The rate of change of $S_{\mathrm{dyn}}$ becomes

$$
\begin{gathered}
\dot{S}_{\mathrm{dyn}}=-\sqrt{\tilde{v}_{2}^{2}+\tilde{v}_{3}^{2}}\left[\sin \alpha C_{D}(\alpha)+\cos \alpha C_{L}(\alpha)\right] f_{0}(\boldsymbol{v}) \\
-k_{v_{1}} \tilde{v}_{1}^{2}+\tilde{\boldsymbol{\omega}} \cdot \boldsymbol{u}+\tilde{\boldsymbol{\omega}} \cdot \boldsymbol{\tau}_{v} \leq \tilde{\boldsymbol{\omega}} \cdot \boldsymbol{u}
\end{gathered}
$$

The dissipation inequality above suggests that the subsystem (10) and (11) has been rendered passive with input $\boldsymbol{u}$ and output $\boldsymbol{y}=\tilde{\boldsymbol{\omega}}$. Of course, recalling Remark 2.2, the notion of passivity for systems defined by discontinuous equations requires some care. Regardless, one may conclude from Theorem 1 on p. 153 of [14] that the origin is a stable equilibrium of the system (10) and (11) when $\boldsymbol{u}=\mathbf{0}$. Moreover, one may use an extension of Lasalle's invariance principle presented in [15] to conclude asymptotic stability. At this point, we have completed the first step of a two-step design procedure in which we first stabilize the vehicle dynamics and then the attitude kinematics.

Remark 3.1: Although Assumption 2.3 concerning the form of $C_{D}(\alpha)$ and $C_{L}(\alpha)$ is quite mild, the thrust control law (12) assumes precise knowledge of these functions. One can improve robustness to uncertainty in these coefficients by instead choosing

$$
f_{c}=-\kappa \operatorname{sgn}\left(\tilde{v}_{1}\right) f_{0}(\boldsymbol{v})-k_{v_{1}} \tilde{v}_{1}
$$

where

$$
\kappa>\sup _{\alpha}\left|\cos \alpha C_{D}(\alpha)-\sin \alpha C_{L}(\alpha)\right|
$$

Of course, the signum function introduces another discontinuity into the system dynamics. Such discontinuities appear in certain robust nonlinear control methods, such as sliding mode control. 
Implementation difficulties, such as chattering behavior, can be avoided by replacing the discontinuous signum function with a smooth approximation. See [17], for example.

Remark 3.2: Referring to ( $\overline{15})$, note that one could accommodate angular rate dependency in the viscous force $f_{v}$, provided $\omega$ appears as follows:

$$
\boldsymbol{f}_{v}(\boldsymbol{v}, \boldsymbol{\omega})=\boldsymbol{f}_{v_{v}}(\boldsymbol{v})+\boldsymbol{F}_{v_{\omega}}(\boldsymbol{v}, \boldsymbol{\omega}) \boldsymbol{\omega}
$$

where $\boldsymbol{f}_{v_{v}}$ takes the form (8) and where the components of the $3 \times 3$ matrix $\boldsymbol{F}_{v_{\omega}}(\boldsymbol{v}, \boldsymbol{\omega})$ are known.

The rotational error kinematics (9) are passive with input $\tilde{\boldsymbol{\omega}}$, output $\boldsymbol{e}_{\chi}$, and storage function

$$
S_{\mathrm{kin}}(\tilde{\boldsymbol{e}})=\frac{1}{2} k_{e} \tilde{\boldsymbol{e}} \cdot \tilde{\boldsymbol{e}}
$$

where $k_{e}>0$; see [18], for example. Note that

$$
S_{\mathrm{kin}}(\tilde{\boldsymbol{e}})=\frac{1}{2} k_{e}\left[\left(1-e_{0}\right)^{2}+\boldsymbol{e}_{\chi} \cdot \boldsymbol{e}_{\chi}\right]=k_{e}\left(1-e_{0}\right)
$$

The feedback passivation technique described in Sec. 4.3 of [ 8 ] suggests choosing

$$
\boldsymbol{u}=\boldsymbol{w}-\left[\frac{\partial S_{\text {kin }}}{\partial \tilde{\boldsymbol{e}}} \cdot\left(\frac{1}{2} \boldsymbol{Q}\left(\tilde{\boldsymbol{e}}+\boldsymbol{e}_{d}\right)\right)\right]^{T}=\boldsymbol{w}-\frac{1}{2} k_{e} \boldsymbol{e}_{\chi}
$$

Keeping in mind the technical implications of Remark 2.2, the control law given above would appear to render the complete closedloop system passive from the new input $\boldsymbol{w}$ to the output $\boldsymbol{y}=\tilde{\boldsymbol{\omega}}$ with respect to the positive definite storage function

$$
S(\tilde{\boldsymbol{e}}, \tilde{\boldsymbol{p}}, \tilde{\boldsymbol{h}})=S_{\mathrm{kin}}(\tilde{\boldsymbol{e}})+S_{\mathrm{dyn}}(\tilde{\boldsymbol{p}}, \tilde{\boldsymbol{h}})
$$

To see this, observe that

$$
\begin{aligned}
\dot{S}= & -\sqrt{\tilde{v}_{2}^{2}+\tilde{v}_{3}^{2}}\left[\sin \alpha C_{D}(\alpha)+\cos \alpha C_{L}(\alpha)\right] f_{0}(\boldsymbol{v})-k_{v_{1}} \tilde{v}_{1}^{2} \\
& +\boldsymbol{\tau}_{v} \cdot \tilde{\boldsymbol{\omega}}+\boldsymbol{y} \cdot \boldsymbol{w} \leq \boldsymbol{y} \cdot \boldsymbol{w}
\end{aligned}
$$

Apart from any concern over passivity for discontinuous systems, one may recognize that $S$ is a Lyapunov function and that choosing $\boldsymbol{w}=-\boldsymbol{K} \tilde{\boldsymbol{\omega}}$ with $\boldsymbol{K}>0$ ensures that $\dot{S} \leq 0$. To conclude global asymptotic stability, note that $S$ is radially unbounded, and apply Lasalle's invariance principle, as extended for nonsmooth systems [15]. Define the set

$$
E=\{(\tilde{\boldsymbol{e}}, \tilde{\boldsymbol{p}}, \tilde{\boldsymbol{h}}) \mid \dot{S}=0\}
$$

and let $\mathcal{M}$ be the largest invariant set contained in $E$. By Lasalle's principle, trajectories converge to the set $\mathcal{M}$ as $t \rightarrow \infty$. If $\mathcal{M}=\{\mathbf{0}\}$, then the equilibrium is globally asymptotically stable. Now, $\dot{S} \equiv 0$ implies that $\boldsymbol{v} \equiv \boldsymbol{v}_{d}$ and that $\boldsymbol{\omega} \equiv \mathbf{0}$. This implies that $\boldsymbol{p} \equiv \boldsymbol{p}_{d}$ and $\boldsymbol{h} \equiv \boldsymbol{h}_{d}$. Thus, within the set $\mathcal{M}$,

$$
\dot{\tilde{\boldsymbol{h}}}=\mathbf{0}=-\frac{1}{2} k_{e} \boldsymbol{e}_{\chi}
$$

It follows that $\boldsymbol{e}_{\chi}=\mathbf{0}$ which implies that $e_{0}=1$ and $\tilde{\boldsymbol{e}}=\mathbf{0}$.

Theorem 3.3: The feedback control law

$$
f_{c}=f_{0}(\boldsymbol{v})\left[\cos \alpha C_{D}(\alpha)-\sin \alpha C_{L}(\alpha)\right]-k_{v_{1}} \tilde{v}_{1}
$$

and

$$
\boldsymbol{\tau}_{c}=-\left[\boldsymbol{r}_{\mathrm{cm}} \times m g\left(\boldsymbol{R}_{\mathrm{IB}}^{T} \boldsymbol{i}_{3}\right)+\boldsymbol{p} \times \boldsymbol{v}_{d}\right]-\boldsymbol{K} \boldsymbol{\omega}-\frac{1}{2} k_{e} \boldsymbol{e}_{\chi}
$$

globally asymptotically stabilizes the steady motion

$$
\boldsymbol{e}=\left(\begin{array}{l}
1 \\
0 \\
0 \\
0
\end{array}\right), \quad \boldsymbol{v}=\left(\begin{array}{c}
v_{d} \\
0 \\
0
\end{array}\right), \quad \boldsymbol{\omega}=\left(\begin{array}{l}
0 \\
0 \\
0
\end{array}\right)
$$

with $v_{d}>0$ provided

$$
k_{e}>0, \quad k_{v_{1}}>0, \quad \text { and } \quad K>0
$$

Remark 3.4: One may modify the control law proposed in Theorem 3.3, using a simple line-of-sight scheme, to drive the vehicle to a desired linear path in inertial space. This is a special case of the more general (and more difficult) problem of threedimensional path following. Recall that the control law given by (17) and (18) stabilizes steady longitudinal translation in a particular inertial direction. To stabilize motion along a line, one might vary the desired orientation, expressed by $\boldsymbol{q}_{d}$, in a way that causes the vehicle's trajectory to converge to the line.

Considering only linear paths, one may assume without further loss of generality that the desired path coincides with the positive inertial $\boldsymbol{i}_{1}$ axis. Let $\boldsymbol{\mu}$ be the vector (expressed in the inertial frame) from the body frame origin to a point on the $\boldsymbol{i}_{1}$ axis which is some "look-ahead distance" $k_{x} L$ further along the line:

$$
\boldsymbol{\mu}=\left(\begin{array}{c}
x(1)+k_{x} L \\
0 \\
0
\end{array}\right)-\boldsymbol{x}=\left(\begin{array}{c}
k_{x} L \\
-x(2) \\
-x(3)
\end{array}\right)
$$

The vector $\boldsymbol{\mu}$ provides coordinates for the translational kinematics normal to the $\boldsymbol{i}_{1}$ axis. [Note that the along-track position $x(1)$ is ignored]. The translational kinematics are

$$
\dot{\mu}=-\left(\mathbb{1}-\boldsymbol{\beta}_{1} \boldsymbol{\beta}_{1}^{T}\right) \boldsymbol{R}_{\mathrm{IB}}(\boldsymbol{q}) \boldsymbol{v}
$$

The desired orientation, which depends on the translational error, may be obtained by using the vector $\boldsymbol{\mu}$ to define the desired attitude matrix $\boldsymbol{R}_{\mathrm{IB}}\left(\boldsymbol{q}_{d}\right)$. (In this case, if the vehicle assumes the desired attitude, the $\boldsymbol{b}_{1}$ axis is directed toward a point some distance $k_{x} L$ ahead on the $\boldsymbol{i}_{1}$ axis.)

Of course, this modification of the directional stabilization algorithm violates the stability analysis supporting Theorem 3.3, since $\boldsymbol{q}_{d}$ now varies with the system state. A more involved stability analysis is required to show global asymptotic stability to the desired linear path. For a simple AUV model, spectral analysis verifies that the approach works locally, provided the conditions of Theorem 3.3 are met and provided $k_{x}>0$. Simulations suggest that the proposed line-following algorithm provides global asymptotic stability, but this claim has not been proven.

\section{Conclusions}

Feedback passivation was used to develop a nonlinear feedback control law which globally asymptotically stabilizes longitudinalaxis translation of a slender AUV in a desired inertial direction. Because the hydrodynamic angles which transform the viscous force into the body reference frame are discontinuous at the desired equilibrium, the analysis required the use of extended versions of Lyapunov's direct method and Lasalle's invariance principle to systems with discontinuous equations. The stability result was proven using very general assumptions about the viscous force and moment, allowing for a broad range of global, viscous models.

\section{Acknowledgements}

This work was supported in part by NSF grant CMS-0133210, ONR grant N00014-01-1-0588, and ONR grant N00014-05-1-0516. The authors gratefully acknowledge Naira Hovakimyan and Vahram Stepanyan for several enlightening discussions.

\section{References}

[1] Astolfi, A., Chhabra, D., and Ortega, R., "Asymptotic Stabilization of Selected Equilibria of the Underactuated Kirchhoff's Equations," Proceedings of the Americal Control Conference, IEEE, Piscataway, NJ, 2001, pp. 4862-4867.

[2] Leonard, N. E., "Stabilization of Underwater Vehicle Dynamics with Symmetry-Breaking Potentials," Systems and Control Letters, Vol. 32, No. 1, 1997, pp. 35-42.

[3] Encarnaçao, P., and Pascoal, A., "3D Path Following for Autonomous Underwater Vehicle," Proceedings of the IEEE Conference on 
Decision and Control, IEEE, Piscataway, NJ, 2000, pp. 29772982.

[4] Do, K. D., and Pan, J., "Robust and Adaptive Path Following for Underactuated Autonomous Underwater Vehicles," Proceedings of the American Control Conference, IEEE, Piscataway, NJ, 2003, pp. 19941999.

[5] Aguiar, A. P., and Hespanha, J. P., "Logic-Based Switching Control for Trajectory-Tracking and Path-Following of Underactuated Autonomous Vehicles with Parametric Modeling Uncertainty," Proceedings of the Americal Control Conference, IEEE, Piscataway, NJ, 2004, pp. 3004-3010.

[6] Skjetne, R., Fossen, T. I., and Kokotovic, P. V., "Robust Output Maneuvering for a Class of Nonlinear Systems," Automatica, Vol. 40, No. 3, March 2004, pp. 373-383.

[7] Kim, H.-Y., and Woolsey, C. A., "Directional Control of a Streamlined Underwater Vehicle by Feedback Passivation," Proceedings of the Americal Control Conference, IEEE, Piscataway, NJ, 2004, pp. 2998 3003.

[8] Sepulchre, R., Jankovic, M., and Kokotović. P., Constructive Nonlinear Control, Springer-Verlag, London, 1997.

[9] Murray, R. M., Li, Z., and Sastry, S. S., A Mathematical Introduction to Robotic Manipulation, CRC Press, Ann Arbor, MI, 1994.
[10] Fjellstad, O.-E., and Fossen, T. I., "Position and Attitude Tracking of AUV's: A Quaternion Feedback Approach,” IEEE Journal of Oceanic Engineering, Vol. 19, No. 4, 1994, pp. 512-518.

[11] Lamb, H., Hydrodynamics, 6th ed., Dover, New York, 1932.

[12] Newman, J. N., Marine Hydrodynamics, The MIT Press, Cambridge, MA, 1977.

[13] Fossen, T. I., Marine Control Systems, Marine Cybernetics, Trondheim, Norway, 2002.

[14] Filippov, A. F., Differential Equations with Discontinuous Righthand Sides, Kluwer Academic Publishers, Boston, MA, 1988.

[15] Shevitz, D., and Paden, B., "Lyapunov Stability Theory of Nonsmooth Systems," IEEE Transactions on Automatic Control, Vol. 39, No. 9, 1994, pp. 1910-1914.

[16] Wetzel, T. G., and Simpson, R. L., "Unsteady Crossflow Separation Location Measurements on a Maneuvering 6:1 Prolate Spheroid," AIAA Journal, Vol. 38, No. 11, 1998, pp. 2063-2071.

[17] Khalil, H. K., Nonlinear Systems, 3rd ed., Prentice-Hall, Englewood Cliffs, NJ, 2002.

[18] Lizarralde, F., and Wen, J. T., "Attitude Control Without Angular Velocity Measurement: A Passivity Approach," Physica D, Vol. 41, No. 3, 1996, pp. 468-472. 